\title{
Absenteísmo na Indústria está Associado com o Trabalho em Turnos e com Problemas no Sono
}

\section{ABSENCE IN INDUSTRY IS ASSOCIATED WITH SHIFT WORK AND WITH PROBLEMS IN SLEEP}

\author{
Rubian Diego Andrade1, Geraldo Jose Ferrari Junior², Renata Capistrano', Clarissa Stefani Teixeira ${ }^{3}$, Thais Silva Beltrame ${ }^{4}$ \\ Érico Pereira Gomes Felden ${ }^{5}$ \\ 1. Doutorando(a) em Ciências do Movimento Humano do Centro de Ciências da Saúde e do Esporte, Universidade do Estado de Santa Catarina \\ (UDESC), Florianópolis, SC, Brasil.
}

2. Mestrando em Ciências do Movimento Humano do Centro de Ciências da Saúde e do Esporte, Universidade do Estado de Santa Catarina (UDESC), Florianópolis, SC, Brasil.

3. Doutora em Engenharia de Produção. Professora da Universidade Federal de Santa Catarina (UFSC), Florianópolis, SC, Brasil.

4. Doutora em Ciências do Movimento Humano. Professora Titular do Centro de Ciências da Saúde e do Esporte, Universidade do Estado de Santa Catarina (UDESC), Florianópolis, SC, Brasil.

5. Doutor em Educação Física. Professor Adjunto do Centro de Ciências da Saúde e do Esporte, Universidade do Estado de Santa Catarina (UDESC), Florianópolis, SC, Brasil.

\begin{abstract}
RESUMO
Introdução: Cada vez mais as indústrias mantêm seu volume de produção durante as $24 \mathrm{~h}$ horas do dia. Esta reorganização temporal traz repercussões importantes para a saúde do trabalhador. 0 objetivo deste estudo foi analisar os fatores associados ao absenteísmo em trabalhadores de diferentes turnos de uma indústria de grande porte do sul do Brasil. Método: A amostra foi composta por 885 profissionais com média de idade de $31,1(8,5)$ anos de uma indústria do setor de telecomunicação. A variável dependente foi avaliada por meio de faltas ao trabalho nos últimos 12 meses por motivos de saúde. Resultados: A frequência de absenteísmo foi de $16,6 \%$. Os fatores associados ao desfecho foram a idade avançada $(\mathrm{OR}=2,16$; IC95\%= $1,27-3,67)$, o trabalho em turnos extremos, primeiro turno $(\mathrm{OR}=1,91$; IC95\%= 1,08-3,36) e terceiro turno (OR=2,06; IC95\%= 1,01-4,25), percepção elevada de estresse $(\mathrm{OR}=1,74 ; \mathrm{IC} 95 \%=1,07-2,82)$ e os distúrbios do sono $(\mathrm{OR}=2,03$; IC95\%= $1,07-3,76)$. Não foram identificadas associações entre absenteísmo e fatores de estilo de vida como prática de atividade física e consumo de álcool e tabaco. Conclusão: Trabalhadores com distúrbios de sono e que exercem suas funções em turnos extremos (manhã ou noite) possuíram mais chance de faltar ao trabalho por questões de saúde.
\end{abstract}

(Andrade RD, Ferrari Junior GJ, Capistrano R, Teixeira CS, Beltrame TS, Felden ÉPG, 2017. Absenteísmo na Indústria está Associado com o Trabalho em Turnos e com Problemas no Sono. Cienc Trab. Ene-Abr; 19 [58]: 35-41).

Palavras-chave: TRABALHO EM TURNOS, SONO, ABSENTEÍSMO, TRABALHADORES, SAÚDE DO TRABALHADOR.

\section{ABSTRACT}

Introduction: Increasingly the industry maintains its production volume during the 24 hours of the day. This time reorganization has important implications for workers' health The aim of this study was to analyze factors associated with absenteeism among workers in different shifts of a large industry in southern Brazil. Methods: The sample consisted of 885 professionals with an average age of 31.1 (8.5) years of the telecommunication sector industry. The dependent variable was assessed by absence from work in the last 12 months for health reasons. Results: The absenteeism rate was $16.6 \%$. Factors associated with outcome were advanced age $(\mathrm{OR}=2.16,95 \% \mathrm{CI} 1.27$ to 3.67), work in extreme shifts, first round (OR $=1.91$, 95\% CI 1.08 3.36) and third round ( $\mathrm{OR}=2.06,95 \% \mathrm{CI} 1.01$ to 4.25 ), high perceived stress ( $\mathrm{OR}=1.74,95 \% \mathrm{CI} 1.07$ to 2.82 ) and sleep disorders $(\mathrm{OR}=2.03$, 95\% CI 1.07 to 3.76). No associations were found between absenteeism and lifestyle factors such as physical activity and consumption of alcohol and tobacco. Conclusion: Workers with sleep disorders and perform their duties in extreme shifts (morning or night) possessed more likely to miss work for health reasons.

Key words: SHIFT WORK, SLEEP, ABSENTEEISM, WORKERS, OCCUPATIONAL HEALTH.

\author{
Correspondência / Correspondence: \\ Rubian Diego Andrade \\ CEFID/UDESC \\ Rua Paschoal Simoni 358, Florianópolis - SC, Brasil CEP: 88080-350 \\ Telefone: 48-3321-8600 \\ e-mail: rubiandiego@gmail.com \\ Recibido: 27 de Noviembre de 2016 / Aceptado: 04 de Marzo de 2017
}

\section{INTRODUÇÃO}

Uma mudança marcante na organização do trabalho contemporâneo é o funcionamento das indústrias durante as 24 horas do dia gerando a necessidade da organização das atividades em turnos. Este tipo de trabalho caracteriza-se por ser realizado em horário diferente do horário formal (diurno). Pode ser realizado em turnos fixos (manhã, tarde ou noite), rodizios (escalas) ou em ciclos comprimidos (geralmente em empresas petroquímicas), entre oito a 12 horas de duração. ${ }^{1}$ 
Estima-se que na Europa e nos Estados Unidos 13\% a 14\% dos trabalhadores estão inseridos no contexto do trabalho em turnos, especialmente à noite. ${ }^{2}$ Apesar de não haver dados oficiais com relação ao número de trabalhadores nesta modalidade no Brasil, acredita-se que este percentual seja próximo a esse valor ${ }^{1}$. Um dos principais problemas dessa atuação se associa ao que é imposto ao ser humano pelo trabalho executado em turnos, considerando o conflito entre sua natureza diurna e um ritmo artificial de atividade e iluminação. Tal situação pode não ser suportada por muitos indivíduos e aumentam o risco de acometimento de inúmeras patologias. ${ }^{3}$

Por esta razão, os estudos sobre a temática se reportam aos termos tolerância ou resiliência ${ }^{4}$ ao descreverem aqueles possíveis fatores, de ordem social e, especialmente de ordem individual, que estariam associados com menores consequências do trabalho em turnos para a saúde. Esta discussão aponta para dúvidas a respeito da real possibilidade de considerar que o ser humano possa se adaptar de forma plena a este tipo de trabalho.

Neste contexto, questiona-se sobre a influência que as atividades em turnos exercem sobre as faltas ao trabalho principalmente pelos fatores associados à saúde. Também denominada absenteísmo, a falta ao trabalho é considerada importante problema nas organizações. Impacta de forma negativa na produção, em custos com a reposição da mão de obra, bem como na diminuição da qualidade do trabalho. ${ }^{5}$ Teixeira ${ }^{6}$ destaca que os prejuízos em não manter um trabalhador saudável dentro das organizações repercutem, tanto nas questões sociais, quanto nas questões econômicas para o estado, empresa e para o próprio indivíduo.

Em decorrência disso, diferentes classes de trabalhadores foram investigadas no sentido de identificar variáveis que possam se associar à ausência ao trabalho. Os resultados destas pesquisas apontam que fatores demográficos, satisfação com o emprego, características organizacionais, níveis de autonomia e responsabilidade estão associadas ao absenteísmo em trabalhadores de diversos setores da economia. ${ }^{7,8}$ No entanto, a relevância do trabalho em turnos no entendimento do absenteísmo em conjunto com diversos fatores, principalmente em trabalhadores brasileiros, é uma importante análise de ser realizada. Diante disso, este estudo teve como objetivo analisar os fatores associados ao absenteísmo em trabalhadores de diferentes turnos de uma indústria de grande porte do sul do Brasil.

\section{MÉTODO}

\section{Populaçáo e amostra}

A população considerada para este estudo foi de 1.674 trabalhadores industriários do segmento de telecomunicações (destes 720 trabalham na produção e 954 em setores administrativos) de ambos os sexos, contratados sob o regime celetista de uma das indústrias de grande porte da Grande Florianópolis. Para o cálculo amostral foram considerados os seguintes parâmetros: prevalência de 50\% para desfechos desconhecidos, erro tolerável de amostragem de 4\% com nível de confiança de 95\% e previsão de perdas de 20\%, resultando numa amostra mínima de 532 sujeitos. ${ }^{9}$ Participaram do estudo 885 trabalhadores industriários da região da Grande Florianópolis-SC, Brasil. A empresa foi escolhida por ser uma das únicas da região que opera em regime de turno de trabalho, incluindo o trabalho noturno.

Os trabalhadores foram convidados a participarem da pesquisa por meio de comunicação interna coletiva e não individual. Assim, todos tiveram as mesmas oportunidades de fazer parte da pesquisa. Este procedimento foi realizado para facilitar o processo de coleta de dados em um ambiente corporativo e minimizar possível viés de seleção amostral. Os trabalhadores assinaram o Termo de Consentimento Livre e Esclarecido. A coleta de dados ocorreu no próprio ambiente de trabalho em momentos pré-agendados com a organização, evitando possíveis perdas amostrais. Para a realização desta pesquisa, foram cumpridos os princípios éticos contidos na Declaração de Helsinki e na Resolução 466/12 do Conselho Nacional de Saúde. Este estudo foi aprovado pelo Comitê de Ética Envolvendo Seres Humanos da Universidade do Estado de Santa Catarina, conforme parecer n ${ }^{\circ}$ 801.409/2014.

\section{Variáveis investigadas}

A variável dependente investigada foi o absenteísmo, analisado de forma dicotômica (sim/não) por meio da questão: "Você já se afastou do trabalho por questões de saúde no último ano?”. Além disso, o número de eventos ocorridos nesse período também foi questionado.

As variáveis independentes investigadas foram o turno, qualidade do sono, percepção de estresse, consumo de álcool e tabaco, nível de atividade física, status de peso além de variáveis sociodemográficas. Os protocolos de análise destas variáveis estão apresentados a seguir.

Considerou-se como primeiro turno os participantes que relataram exercer suas funções entre 5h50min e 14h20min, segundo turno das $14 \mathrm{~h} 10 \mathrm{~min}$ às $22 \mathrm{~h} 37 \mathrm{~min}$, terceiro turno das $22 \mathrm{~h} 27 \mathrm{~min}$ às 6h e turno integral, àqueles que exercem suas funções entre 7h30min e 17h30min.

A qualidade do sono foi avaliada de forma subjetiva em relação ao último mês, pelo questionário Pittsburgh Sleep Quality Index - (PSQI) criado por Buysse et al. ${ }^{10}$ que foi traduzido e validado para o português do Brasil por Bertolazi et al. ${ }^{11}$ A validação brasileira ocorreu de forma concorrente com a polissonografia (medida objetiva) em 83 pacientes e 21 indivíduos do grupo controle. A PSQI brasileira mostrou-se válida e confiável para manejo clínico ou pesquisa. Apresentou coeficiente de confiabilidade ( $\alpha$ de Cronbach) de 0,82, indicando um alto grau de consistência interna. 0 questionário consiste de 19 questões auto administradas e cinco questões respondidas pelos seus companheiros de quarto. Estas últimas são utilizadas apenas para informações clínicas e não fizeram parte deste estudo. As questões são agrupadas em sete componentes: 1) qualidade subjetiva do sono; 2) latência do sono; 3) duração do sono; 4) eficiência habitual do sono; 5) distúrbios do sono; 6) uso de medicação para dormir; 7) sonolência diurna e distúrbios durante o dia. Os pesos são distribuídos numa escala de zero (0) a três (3). As pontuações desses componentes são somadas para produzirem uma pontuação geral, que varia de zero a 21, no qual quanto maior a pontuação, pior a qualidade do sono. Escores superiores a cinco indicam qualidade ruim de sono e acima de dez indicam a presença de distúrbios do sono.

A percepção de estresse foi avaliada a partir da questão: "Como você descreve o nível de estresse em sua vida? ”. Para esta variável considerou-se como percepção de baixo estresse as alternativas "raramente estressado" e "às vezes estressado" e, como percepção de alto estresse as alternativas "quase sempre estressado" e "excessivamente estressado". ${ }^{12}$

0 consumo de bebidas alcoólicas foi analisado a partir da per- 
gunta: "Quantas doses de bebida alcoólica você toma em uma semana normal? ". Considerando como uma dose, 1/2 garrafa de cerveja, um copo de vinho ou uma dose de uísque, conhaque, cachaça ou vodca. Considerou-se como baixo consumo àqueles participantes que relataram consumir nenhuma a sete doses semanais e, alto consumo valores superiores a oito doses de bebidas alcoólicas por semana. Já para a variável tabagismo considerou-se como "não fumante" àqueles que responderam ser "fumante ocasional", "exfumante" ou "não fumante". E "fumantes" àqueles que assumiram fazer uso de cigarros regularmente. ${ }^{13}$

0 status de peso foi calculado a partir dos valores autorreferidos de massa corporal e altura com o cálculo do Índice de Massa Corporal (IMC) e categorizado de acordo com os pontos de corte da Organização Mundial da Saúde (OMS) em eutróficos (IMC $\leq 24,9 \mathrm{~kg}$ / $\mathrm{m}^{2}$ ) e sobrepeso/obesidade (IMC $\left.\geq 25,0 \mathrm{~kg} / \mathrm{m}^{2}\right) .{ }^{14}$

0 nível de atividade física foi avaliado por meio do Questionário Internacional de Atividade Física (IPAQ - versão curta), elaborado com suporte da Organização Mundial da Saúde e validado no Brasil pelo Centro de Estudos do Laboratório de Aptidão Física de São Caetano do Sul (CELAFISCS).$^{15}$ Este instrumento leva em consideração o tempo dispendido em atividades físicas moderadas e vigorosas nos últimos sete dias em uma semana habitual. Para esta análise foram considerados insuficientemente ativos àqueles trabalhadores com menos de 150 minutos em atividades físicas moderadas e vigorosos, e, ativos àqueles que obtiveram tempo superior a 150 minutos durante a última semana.

Com relação às questões sociodemográficas, o questionário continha perguntas quanto à idade (que para fins de análise foi categorizada em até 29,9 anos, de 30 a 39,9 anos e acima de 40 anos) e renda familiar em reais $(\mathrm{R} \$$ ) categorizada por meio de tercis em baixa, média e alta renda.

\section{Análise estatística}

Foram realizadas análises descritivas (médias, frequências e desvios -padrões). Para as análises inferenciais, utilizou-se o teste U de Mann-Whitney para comparação das médias entre os grupos e o teste Qui-quadrado para associação entre as variáveis categóricas por meio do programa estatístico SPSS 20.0. Além disso, recorreu-se a Regressão Logística Binária com o objetivo de testar as hipóteses de associação das variáveis sociodemográficas (sexo, faixa etária, turno de trabalho e renda) e de saúde (percepção de estresse, tabagismo, consumo de bebidas alcóolicas, status de peso, nível de atividade física e qualidade do sono) sobre o absenteísmo. Foram analisados modelos brutos e considerados para os modelos ajustados as variáveis com $\mathrm{p} \leq 0,20^{16}$ no teste de Qui-quadrado. Para todas as demais análises considerou-se um nível de significância de 5\%.

\section{RESULTADOS}

A média de idade dos trabalhadores investigados foi de 31,1 anos. De modo geral, a maior parte da amostra $(58,2 \%)$ foi composta por mulheres e pertencentes ao turno integral de trabalho (73,3\%). Ainda com relação à análise descritiva, a proporção do sexo feminino foi superior no turno geral de trabalho $(\mathrm{p}<0,001)$, bem como na categoria renda familiar baixa $(\mathrm{p}<0,001)$.

Ao analisar as variáveis de saúde na amostra geral, identificou-se que a maior parte dos trabalhadores (83,6\%) apresentou baixa percepção de estresse. Grande parte (95,8\%) declarou-se não fumantes e com nenhum ou pouco consumo de bebidas alcóolicas por semana (96,3\%).
Identificou-se ainda, predomínio de trabalhadores eutróficos (55,9\%), ativos fisicamente $(60,7 \%)$ e com qualidade de sono ruim $(54,1 \%)$.

Ao comparar as variáveis de saúde entre os sexos, diferenças estatisticamente significativas foram observadas nas variáveis, consumo de álcool $(\mathrm{p}<0,001)$, status de peso $(\mathrm{p}<0,001)$ e nível de atividade física $(\mathrm{p}<0,001)$. Cabe destacar que o sexo masculino apresentou maior índice de alto consumo de álcool $(84,8 \%)$ e de excesso de peso (50,9\%). Já no sexo feminino observou-se maior frequência de nível insuficiente de atividade física (66,4\%) (Tabela 1).

Entre os trabalhadores analisados, 147 (58 homens e 89 mulheres) relataram ter sofrido algum problema de saúde que culminou no seu afastamento do trabalho durante o último ano. Isto representa 16,6\% da amostra total. A Figura 1 representa o percentual de absenteísmo estratificado por sexo. Ao comparar a proporção entre as mulheres faltantes com a proporção dos homens faltantes, não foi identificada diferença significativa ( $\mathrm{p}=0,977)$. A média de ocorrências de faltas ao trabalho por motivos de saúde foi de 1,27 com desvio-padrão de 0,79, com no máximo de seis casos relatados no último ano.

$\mathrm{Na}$ análise dos fatores associados ao absenteísmo verificou-se que os individuos com idade acima de 40 anos apresentaram duas vezes mais chance de faltar no trabalho por questões de saúde em compa-

Tabela 1.

Dados descritivos da amostra estratificados por sexo.

\begin{tabular}{|c|c|c|c|c|}
\hline \multirow[t]{2}{*}{ Variáveis } & \multirow[t]{2}{*}{ Geral } & \multicolumn{2}{|c|}{ Sexo } & \multirow[t]{2}{*}{$\mathrm{p}$-valo } \\
\hline & & Masculino & Feminino & \\
\hline Idade, anos & $31,1(8,5)$ & $30,9(8,5)$ & $31,3(8,5)$ & 0,437 \\
\hline \multicolumn{5}{|l|}{ Faixa etária, \% } \\
\hline Até 29,9 & 46,9 & 43,2 & 56,8 & \multirow{3}{*}{0,775} \\
\hline De 30 a 39,9 & 36,7 & 41,2 & 58,8 & \\
\hline Acima de 40 & 16,4 & 40,3 & 59,7 & \\
\hline \multicolumn{5}{|l|}{ Turno de trabalho, $\%$} \\
\hline Turno integral & 73,3 & 37,9 & 62,1 & \multirow{4}{*}{$<0,001$} \\
\hline Primero turno & 11,4 & 47,5 & 52,5 & \\
\hline Segundo turno & 9,5 & 45,2 & 54,8 & \\
\hline Terceiro turno & 5,8 & 74,5 & 25,5 & \\
\hline \multicolumn{5}{|l|}{ Renda familiar, \% } \\
\hline Baixa & 38,8 & 23,6 & 76,4 & \multirow{3}{*}{$<0,001$} \\
\hline Média & 32,1 & 42,2 & 57,8 & \\
\hline Alta & 29,1 & 64,9 & 35,1 & \\
\hline \multicolumn{5}{|l|}{ Percepção de estresse, \% } \\
\hline Alto & 16,4 & 35,9 & 64,1 & \multirow{2}{*}{0,065} \\
\hline Baixo & 83,6 & 43,0 & 57,0 & \\
\hline \multicolumn{5}{|l|}{ Tabagismo, \% } \\
\hline Fumante & 4,2 & 54,1 & 45,9 & \multirow{2}{*}{0,130} \\
\hline Não fumante & 95,8 & 41,5 & 58,5 & \\
\hline \multicolumn{5}{|c|}{ Consumo de bebidas alcoólicas, $\%$} \\
\hline Baixo consumo & 96,3 & 40,1 & 59,9 & \multirow{2}{*}{$<0,001$} \\
\hline Alto consumo & 3,7 & 84,8 & 15,2 & \\
\hline \multicolumn{5}{|l|}{ Status de peso, \% } \\
\hline Eutróficos & 55,9 & 34,4 & 65,6 & \multirow{2}{*}{$<0,001$} \\
\hline Excesso de peso & 44,1 & 50,9 & 49,1 & \\
\hline \multicolumn{5}{|l|}{ Nível de Atividade Fisica, \% } \\
\hline Ativo & 60,7 & 47,1 & 52,9 & \multirow{2}{*}{$<0,001$} \\
\hline Insuficientemente ativo & 39,3 & 33,6 & 66,4 & \\
\hline \multicolumn{5}{|l|}{ Qualidade do sono } \\
\hline Boa qualidade & 34,6 & 42,6 & 57,4 & \multirow{3}{*}{0,606} \\
\hline Qualidade ruim & 54,1 & 44,1 & 55,9 & \\
\hline Distúrbios do sono & 11,4 & 38,5 & 61,5 & \\
\hline
\end{tabular}

p-valor do teste $U$ de Mann-Whitney para variáveis contínuas ou do Qui-Quadrado para variáveis categorizadas. 
ração com indivíduos mais jovens (OR=2,16; IC95\%: 1,27-3,67). Além disso, trabalhadores noturnos e do primeiro turno, apresentaram duas vezes mais chance de faltar ao trabalho por questões de saúde em relação aos trabalhadores de turno geral $(\mathrm{OR}=2,06$; IC95\%:1,01-4,25; OR=1,91; IC95\%:1,08-3,36). Da mesma forma, indivíduos com alta percepção de estresse (OR=1,74; IC95\%:1,07-

\section{Figura 1.}

Percentual de absenteísmo estratificado por sexo.

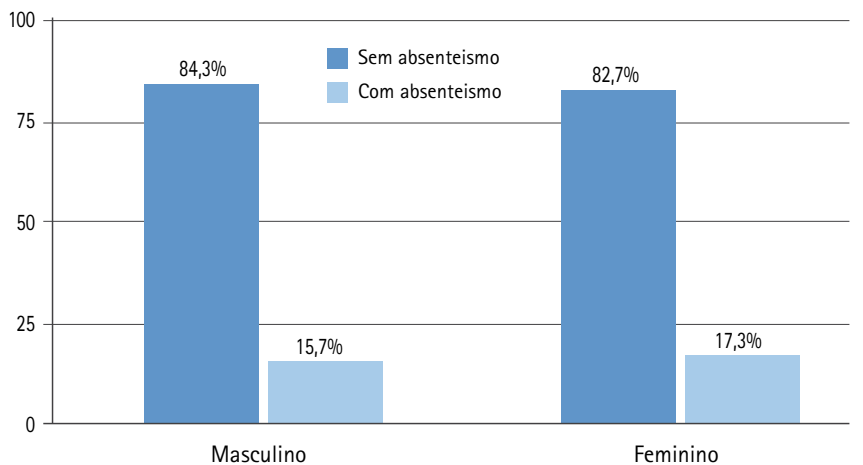

2,82) e com distúrbios do sono (OR=2,03; IC95\%:1,10-3,76) possuiam maior chance de se afastarem do trabalho em comparação com indivíduos com baixa percepção de estresse e boa qualidade de sono respectivamente (Tabela 2).

\section{DISCUSSÃO}

A taxa de absenteísmo é um importante indicador utilizado para mensurar as condições e qualidade de vida no trabalho. Os prejuízos econômicos causados às empresas e à previdência social por conta das faltas e afastamentos ao trabalho são muitos. Por isso, o investimento em questões relacionadas à saúde do trabalhador não deve ser tratado como despesa. É preciso fazer parte da rotina das organizações ações que promovam a qualidade de vida e que melhorem as condições de saúde e segurança no trabalho. ${ }^{17}$ Apesar de o Brasil possuir uma legislação completa que estabelecem diretrizes e recomendações para empresas no que tange as questões de Saúde e Segurança do Trabalhador, segundo dados do Ministério do Trabalho e Previdência Social, os índices de faltas ao trabalho e afastamentos das funções laborais aumentam a cada ano. ${ }^{18}$

Tabela 2. Fatores sociodemográficos, de trabalho e saúde associados ao absenteísmo.

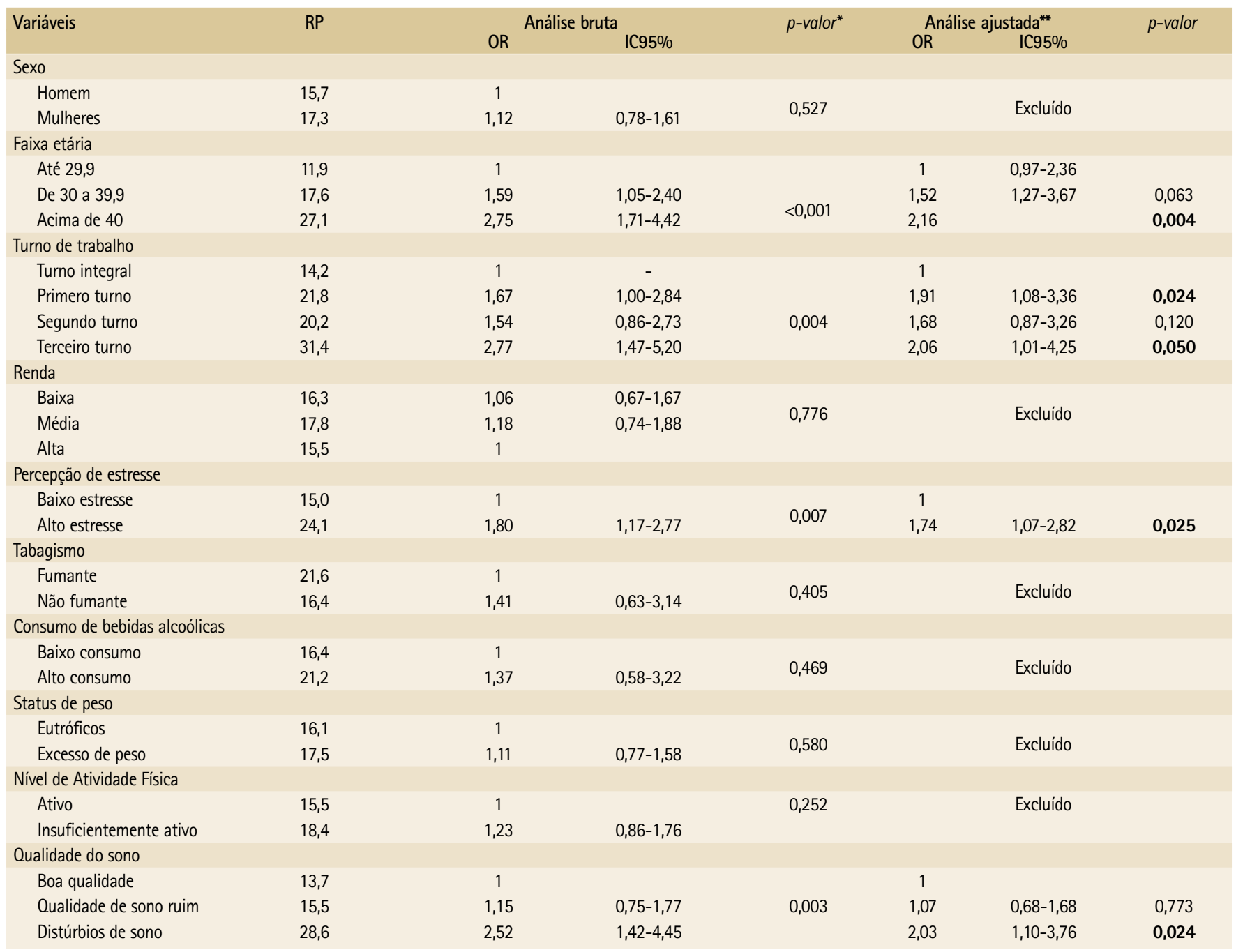

*p-valor do Qui-Quadrado. ${ }^{*}$ Análise ajustada pelas variáveis com $p \leq 0,20$ na análise bruta. 
0 presente estudo não se restringiu a analisar os afastamentos provindos de acidentes de trabalho. Considerou-se como absenteísmo qualquer falta ao trabalho por motivos de saúde no último ano. Assim, esta variável obteve uma maior abrangência, podendo estar relacionada com diversas causas, como por exemplo, dores musculoesqueléticas, problemas dentários, consultas, licenças médicas, acidentes de trabalho, entre outras. Além disso, não foi meta do presente estudo analisar o período no qual o trabalhador permaneceu sem comparecer ao trabalho. De qualquer maneira, a frequência de faltas ao trabalho por motivos de saúde foi baixa (cerca de 17\%) ao considerarmos outras categorias profissionais. Em profissionais da saúde, por exemplo, este percentual foi de $25 \%{ }^{19}$ e, em professores $38 \% .{ }^{20} \mathrm{Na}$ Polônia, estudo conduzido com 11 empresas totalizando 350 trabalhadores, 19\% indicaram que problemas de saúde haviam causado ausência ao trabalho nos últimos 12 meses. ${ }^{21}$ A equivalência do índice encontrado na presente investigação com países desenvolvidos, como no caso da Polônia, sugere a boa qualidade dos serviços de saúde e segurança da empresa analisada, que há anos vem sendo reconhecida como uma das 150 melhores empresas para se trabalhar no Brasil. ${ }^{22}$

Apesar disso, 28,6\% dos trabalhadores apresentaram distúrbios do sono. A espécie humana como um ser diurno possui o seu ciclo vigília-sono como a maioria dos mamíferos, ou seja, durante o dia mantem-se em vigília e a noite necessita de horas de sono como forma de promover a organização dos processos fisiológicos. Os mecanismos de regulação entre o sono e a vigília são sincronizados por agentes endógenos (ritmicidade biológica) e exógenos (ambientais), que no caso dos humanos, os eventos sociais como o trabalho podem ser considerados nesse contexto. Assim, a alteração dos horários de sono pelo trabalho, como no caso dos trabalhadores noturnos, ocasiona mudanças significativas nos mecanismos de consolidação das memórias e na liberação de hormônios, como o cortisol e a melatonina. ${ }^{23}$

De fato, o trabalho em turnos vem sendo associado a diversas enfermidades, entre elas destaca-se o ganho de peso corporal e risco metabólico aumentado ${ }^{24}$, depressão $0^{25}$, câncer de próstata ${ }^{26,27}$ e de mama. ${ }^{27,28}$ Especialmente entre trabalhadores noturnos, recente pesquisa, constatou diminuição considerável do desempenho funcional no local de trabalho gerado pelo cansaço e fadiga. ${ }^{29}$ Assim, em um primeiro momento, os trabalhadores noturnos apresentam tolerância quanto a inversão do ciclo vigília/ sono. No entanto, esse comportamento em longo prazo poderá trazer consequências para a saúde. ${ }^{27,30}$ De acordo com a International Agency for Research on Cancer $^{27}$, o trabalho em turnos faz parte do Grupo 2A de fatores de risco para o câncer. Este grupo inclui agentes cuja associação com o câncer em humanos é provável e já foi confirmada em experimentos com animais.

Todos esses problemas podem anteceder o absenteísmo, e, em eventos mais extremos, ser determinantes em casos de acidentes no trabalho. Segundo as análises realizadas nesse estudo, o risco de faltar ao trabalho por motivos de doença aumenta consideravelmente se os trabalhadores exercem suas funções no primeiro e último turno quando comparados ao turno integral. Nesse sentido, a atenção aos trabalhadores em turnos merece maior intensificação.

Ainda referindo-se ao ciclo vigília/sono, os trabalhadores com distúrbios do sono apresentaram duas vezes mais chances de faltar ao trabalho em comparação aos trabalhadores com boa qualidade do sono. Em concordância com a atual pesquisa, estudos realizados na França $^{31}$, Japão ${ }^{32}$, além de uma revisão sistemática ${ }^{33}$ apontam que a má qualidade e distúrbios do sono estão associados ao absenteísmo.
Por conta de noites mal dormidas, os problemas do sono promovem eventos de sonolência diurna excessiva e fadiga ${ }^{29}$, que prejudicam tanto a saúde e o bem-estar do trabalhador, quanto no rendimento do trabalho.

Nesse sentido, tanto o trabalho em turnos extremos (primeiro e terceiro), quanto os distúrbios do sono, tem repercussões importantes na percepção de estresse e consequentemente impactam na falta ao trabalho por motivos de doença. Resultados semelhantes foram verificados em outros estudos brasileiros com trabalhadores de diversas áreas. ${ }^{34,35}$ Essas pesquisas reforçam as estimativas internacionais da European Agency for Safety and Health at Work. Segundo esta organização, mais de 40 milhões de dias de trabalho são perdidos a cada ano no Reino Unido em razão do estresse, assim como cerca de 225 milhões de dias por ano são perdidos nos Estados Unidos pelo mesmo motivo. ${ }^{36}$ Esse cenário mostra evidências de que o estresse pode ser considerado uma epidemia mundial. Supõe-se que, o número elevado de situações estressantes, aliado à pressão de produtividade em que os trabalhadores estão submetidos diariamente, ocasiona eventos agudos de estresse, que em longo prazo podem tornar-se patológi$\cos ^{12,35}$ Assim, observa-se a necessidade de maior atenção quanto à identificação dos fatores estressores para elaboração de estratégias a fim de prevenir e controlar o estresse. ${ }^{37}$

Esses fatores são potencializados com o avanço da idade, visto que na medida em que o indivíduo envelhece torna-se mais sensivel aos eventos adversos ocasionados pelo trabalho. Nesse sentido, verificouse que os individuos com idade acima de 40 anos apresentaram duas vezes mais chance de faltar no trabalho por questões de saúde em comparação com indivíduos mais jovens. Esse resultado foi semelhante a outros estudos brasileiros realizados com diferentes classes trabalhadoras como, por exemplo, o realizado com enfermeiros ${ }^{38} \mathrm{e}$ servidores públicos. ${ }^{39}$ Também foram encontrados resultados semelhantes em estudos internacionais, como o realizado na Índia com trabalhadores de um hospital ${ }^{40}$ e na Holanda ${ }^{41}$ em estudo de coorte realizado também com trabalhadores industriários. Esses resultados mostram uma relação positiva entre a idade avançada e o absenteísmo, que pode ser justificado pelo processo de envelhecimento, o qual acarreta maior fragilidade do organismo e vulnerabilidade às doenças, diminuindo assim, a capacidade funcional do indivíduo. ${ }^{42-45}$ Com o objetivo de minimizar tais prejuizos algumas empresas buscam readequar o trabalhador em outra função. ${ }^{46}$

Contudo, atenta-se para importância e o cuidado que as empresas devem ter para o trabalhador não se sentir desvalorizado ${ }^{47}$ ou ainda haver desvio de função.

Outro importante resultado identificado no presente trabalho foi a não relação entre o sexo feminino com o absenteísmo. Por muito tempo, a participação de mulheres no mercado de trabalho foi limitada. Isto se deve, entre outros fatores, às licenças no período pósgestacional. No entanto, com a maior participação das mulheres em empregos formais, tais paradigmas estão sendo quebrados. 0 grande número de trabalhadoras na presente amostra fortalece este discurso, e esta análise, promove discussões importantes de igualdade em oportunidades no mundo coorporativo.

Por fim, outras variáveis comportamentais modificáveis também não apresentaram associação com as faltas ao trabalho. Entre elas destaca-se o status de peso, nível de atividade física, consumo de álcool e tabaco. Desta forma, este estudo traz evidências que além das campanhas motivacionais, a organização dos horários de trabalho de acordo com as características de matutinidade e vespertinidade (cronotipo) podem ter maior impacto para a saúde do trabalhador e consequentemente na manutenção de baixos índices de absenteísmo. 


\section{CONCLUSÃO}

0 sono e turno estiveram relacionados às faltas de trabalhadores. Além disso, os industriários com alta percepção de estresse faltaram mais ao trabalho por questões de saúde. Já, com relação às variáveis comportamentais modificáveis, não foi possível estabelecer essa relação. Ainda, observou-se que as mulheres faltaram tanto quanto os homens e o processo de envelhecimento foi um fator importante na relação saúde-doença em trabalhadores.

1. Moreno CRC, Fischer FM, Rotenberg L. A saúde do trabalhador na sociedade 24 horas. São Paulo Perspec. 2003;17(1):34-46.

2. Fullick $S$, Grindey $C$, Edwards $B$, Morris $C$, Reilly $T$, Richardson $D$, et al. Relationships between leisure-time energy expenditure and individual coping strategies for shift-work. Ergonomics. 2009;52(4):448-455.

3. Foster RG, Wulff K. The rhythm of rest and excess. Nat Rev Neurosci. 2005;6(5):407-414.

4. Van Dongen HP, Belenky G. Individual differences in vulnerability to sleep loss in the work environment. Ind health. 2009;47(5):518-526.

5. Baptista PC, Pustiglione M, Almeida MC, Felli VE, Garzin AC, Melleiro MM. Nursing workers health and patient safety: the look of nurse managers. Rev Esc Enferm USP. 2015;49(spe):122-128.

6. Teixeira CS. Saúde e qualidade de vida nos processos de trabalho: um enfoque pertinente para a ergonomia. Ativ Fis Lazer \& Qual Vida: Rev Educ Fis. 2010;1(1):7-25.

7. Kowalczuk K, Krajewska-Kulak E. Influence of selected sociodemographic factors on psychosocial workload of nurses and association of this burden with absenteeism at work. Med Pr. 2015;66(5):615-624.

8. Prestes FC, Beck CL, Magnago TS, Silva RM, Coelho AP. Health problems among nursing workers in a haemodialysis service. Rev gaúcha enferm. 2016;37(1):e50759.

9. Rodrigues PC. Bioestatística. 3a ed. Niterói: EDUFF; 2002.

10. Buysse DJ, Reynolds CF, Monk TH, Berman SR, Kupfer DJ. The Pittsburgh Sleep Quality Index: a new instrument for psychiatric practice and research. Psychiat Res. 1989;28(2):193-213.

11. Bertolazi AN, Fagondes SC, Hoff LS, Dartora EG, Miozzo IC, de Barba ME, et al. Validation of the Brazilian Portuguese version of the Pittsburgh Sleep Quality Index. Sleep med. 2011;12(1):70-75.
12. Pereira ÉF, Teixeira CS, Pelegrini A, Meyer C, Andrade RD, Lopes AdS. Estresse Relacionado ao Trabalho em Professores de Educação Básica. Cienc trab. 2014;16(51):206-210.

13. World Health Organization. Guidelines for the conduct of tobacco-smoking surveys among health professionals; Report of WHO meeting in Collaboration UICC and ACS; 1983 July 7-9; Winnipeg, Canada. Geneva: WHO; 1984.

14. WHO. Obesity: preventing and managing the global epidemic. Geneva: World Health Organization; 2000.

15. Pardini R, Matsudo S, Araújo T, Matsudo V, Andrade E, Braggion G, et al. Validação do questionário internacional de nível de atividade física (IPAQversão 6): estudo piloto em adultos jovens brasileiros. $\mathrm{R}$ Bras $\mathrm{Ci}$ e Mov. 2001;9(3):39-44.

16. Hosmer DW, Lemeshow S, Sturdivant R, editors. Applied Logistic Regression. 3rd ed. New York: Wiley; 2013.

17. Tolbert DV, McCollister KE, LeBlanc WG, Lee DJ, Fleming LE, Muennig P. The economic burden of disease by industry: Differences in quality-adjusted life years and associated costs. Am J Ind Med. 2014;57(7):757-763.

18. Empresa de Tecnologia e Informações da Previdência Social - DATAPREV. Anuário Estatístico de Acidentes de Trabalho-AEAT. Brasilia: Ministerio da Previdência Social; 2013.

19. Mininel VA, Felli VEA, da Silva EJ, Torri Z, Abreu AP, Branco MTA. Cargas de trabalho, processos de desgaste e absenteísmo-doença em enfermagem. Rev Latino-Am Enfermagem. 2013;21(6):1290-1297.

20. Santos MN, Marques AC. Condições de saúde, estilo de vida e caracteristicas de trabalho de professores de uma cidade do sul do Brasil. Ciênc Saúde Coletiva. 2013;18(3):837-846.

21. Pecillo M. Selected aspects of absence at work and work-related health problems in Polish enterprises. Int J occup saf ergo. 2015;21(3):268-275. 
22. As 150 melhores empresas para você trabalhar 2016. Você S.A. [on line]. 2016; (4 out): 00 [citado Jan 2017]. Disponivel em: http://vocesa.uol.com.br/noticias/ mercado/as-150-melhores-empresas-para-voce-trabalhar -2016.phtml

23. Pereira ÉF, Anacleto TS, Louzada FM. Interação entre sincronizadores fóticos e sociais: repercussões para a saúde humana. Rev Biol. 2012;9(3):68-73.

24. Proper KI, van de Langenberg D, Rodenburg W, Vermeulen RC, van der Beek $\mathrm{AJ}$, van Steeg H, et al. The Relationship Between Shift Work and Metabolic Risk Factors: A Systematic Review of Longitudinal Studies. Am j prev med. 2016;50(5):e147-157.

25. Marqueze EC, Vasconcelos S, Garefelt J, Skene DJ, Moreno CR, Lowden A. Natural Light Exposure, Sleep and Depression among Day Workers and Shiftworkers at Arctic and Equatorial Latitudes. Plos One. 2015;10(4): e0122078.

26. Rao D, Yu H, Bai Y, Zheng $X$, Xie L. Does night-shift work increase the risk of prostate cancer? a systematic review and meta-analysis. Oncotargets ther. 2015;8:2817.

27. World Health Organization-WHO. IARC Monographs on the evaluation of carcinogenic risks to humans [on line]. Lion: WHO; 2010 [cited jan 2017]. Available from: http://monographs.iarc.fr/

28. Ijaz S, Verbeek J, Seidler A, Lindbohm M, Ojajarvi A, Orsini N, et al. R. Nightshift work and breast cancer; a systematic review and meta-analysis. Scand J Work Environ Health. 2013;39(5):431-447.

29. De Cordova PB, Bradford MA, Stone PW. Increased errors and decreased performance at night: A systematic review of the evidence concerning shift work and quality. Work. 2016; 53(4):825-834.

30. Fischer FM, Puttonen S, Skene DJ. 21st International symposium on shiftwork and working time: the 24/7 society; from chronobiology to practical life. Chronobiol int. 2014;31(10):1093-1099.

31. Godet-Cayre V, Pelletier-Fleury N, Le Vaillant M, Dinet J, Massuel MA, Leger D. Insomnia and absenteeism at work. Who pays the cost? Sleep. 2006;29(2): 179-184.

32. Doi $Y$, Minowa M, Tango T. Impact and correlates of poor sleep quality in Japanese white-collar employees. Sleep. 2003;26(4):467-471.

33. Kucharczyk ER, Morgan K, Hall AP. The occupational impact of sleep quality and insomnia symptoms. Sleep med rev. 2012;16(6):547-559.

34. Bargas EB, Monteiro MI. Fatores relacionados ao absenteísmo por doença entre trabalhadores de Enfermagem. Acta Paul Enferm. 2014;27(6):533-538.
35. Silva-Junior JS, Fischer FM. Sickness absence due to mental disorders and psychosocial stressors at work. Rev Bras Epidemiol. 2015;18(4):735-744.

36. Cox T, Griffiths $D$, Rial-Conzaléz E. European agency for safety and health at work: research on work-related stress. Luxembourg: Publications Oficce of the European Union; 2000.

37. Benetti C, Magri N, Campos DC, Júnior EG, Camargo ML, Feijó MR. A importância de ações estratégicas de gestão de pessoas no manejo do estresse e de estressores ocupacionais. Omnia Saúde. 2014;11(2):9-24.

38. Ferreira RC, Griep RH, da Fonseca MdJM, Rotenberg L. Abordagem multifatorial do absenteismo por doença em trabalhadores de enfermagem. Rev Saúde Pública. 2012;46(2):259-268.

39. Leão ALdM, Barbosa-Branco $A$, Rassi Neto $E$, Ribeiro CAN, Turchi MD. Absenteísmo-doença no serviço público municipal de Goiânia. Rev bras epidemiol. 2015;18(1):262-277.

40. Darivemula SB, Goswami K, Gupta SK, Salve H, Singh U, Goswami AK. Workrelated Neck Pain Among Desk Job Workers of Tertiary Care Hospital in New Delhi, India: Burden and Determinants. Indian J Community Med. 2016;41(1):50-54.

41. Norder G, Bultmann U, Hoedeman R, de Bruin J, van der Klink JJ, Roelen CA. Recovery and recurrence of mental sickness absence among production and office workers in the industrial sector. Europ J Public Health. 2015;25(3): 419-423.

42. Virtuoso JF, Gregório LPP, Medeiros PAd, Mazo GZ. The" Timed Up and Go" in the prediction and explanation of falls in old people practicing physical exercises. Rev Bras Cineantropom Desempenho Hum. 2014;16(4):381-389.

43. Seitsamo J, Tuomi K, Martikainen R. Activity, functional capacity and wellbeing in ageing Finnish workers. Occup Med. 2007;57(2):85-91.

44. Algarni FS, Gross DP, Senthilselvan A, Battie MC. Ageing workers with workrelated musculoskeletal injuries. Occup Med. 2015;65(3):229-237.

45. Stynen $D$, Jansen NW, Kant IJ. The impact of depression and diabetes mellitus on older workers' functioning. J Psychosom Res. 2015;79(6):604-613.

46. Siukola A, Virtanen $P$, Huhtala $H$, Nygard CH. Absenteeism following a workplace intervention for older food industry workers. Occup Med. 2011; 61(8):583-585.

47. Rodrigues MR, Brêtas ACP. 0 envelhecimento no trabalho na perspectiva de trabalhadores da área de enfermagem. Trab Educ Saúde. 2015;13(2): 343-360. 